\title{
Norteando em sala de aula do ensino médio o ensino sobre Neutrinos via transcrição da Aprendizagem Significativa de Ausubel proposta por Moreira com o emprego da UEPS.
}

\author{
Gomes, F. E. ${ }^{1 *}$; Alvarenga, F. G. ${ }^{2}$ \\ 1 Escola Estadual de Ensino Fundamental e Médio Dionysio Costa, Mutum, MG, Brasil. \\ 2 Departamento de Física, Universidade Federal do Espírito Santo, Vitória, ES, Brasil.
}

* e-mail: harps1925@hotmail.com

\begin{abstract}
Resumo
A investigação em questão está inserida no âmbito da pesquisa qualitativa e quantitativa em ensino de Física. A linha de pesquisa ou sujeito epistêmico serão os Neutrinos. O problema de pesquisa pode ser resumido no seguinte questionamento: como podemos modelar um produto educacional e testá-lo como um método de pesquisa operacional em ensino de Física Contemporânea (FC) para o ensino médio de forma que os alunos aprendam em conformidade com a Teoria da Aprendizagem Significativa (TAS) de David Ausubel? Para a coleta de dados utilizaremos uma Sequência Didática inspirada nas Unidades de Ensino Potencialmente Significativas (UEPS), que são sequências didáticas conforme os pressupostos da teoria retro mencionada. A meta principal da investigação é aplicar a Sequência Didática (SD) em questão aos alunos de duas turmas do terceiro ano do Ensino Médio e verificar sua eficácia como ferramenta pedagógica para o ensino de Neutrinos. Resumidamente, trata-se de uma pesquisa em ensino de Física cuja meta almejada é trazer tópicos de Física Contemporânea para as salas de aula do Ensino Médio
\end{abstract}

\begin{abstract}
The research in question falls within the scope of qualitative and quantitative research in teaching Physics. The line of research or epistemic subject will be Neutrinos. The research problem can be summed up in the following question: how can we model and test an operational research method in teaching Contemporary Physics for high school so that students learn in accordance with David Ausubel Theory of Meaningful Learning? For data collection, we will use a Didactic Sequence inspired by Potentially Significant Teaching Units, which are didactic sequences according to the assumptions of the aforementioned theory. The main goal of the investigation is to apply a Didactic Sequence in question to students in two classes of the third year of High School and verify its effectiveness as a pedagogical tool for teaching Neutrinos. In short, it is a research in teaching Physics whose goal is to bring related Contemporary Physics to the high school classrooms of my school
\end{abstract}

Keywords: Contemporânea, Aprendizagem, Unidades, Neutrinos.

\section{Introdução}

Na maioria das instituições brasileiras de ensino médio a Física ensinada é ainda a Física do final do século XIX. Além da Física Clássica, imprescindível na maior parte dos cursos de engenharias, precisamos contemplar tópicos de Física Contemporânea nas salas de aula de forma que nossos estudantes se mantenham atualizados quanto a novas descobertas científicas e tecnológicas nos limites da Física de fronteira, aprendendo assim novas definições e conceitos e aplicando-os, se for o caso, em sua vivencial. No meu entendimento, é inaceitável que o sistema educacional prive, involuntariamente, nossos estudantes desses avanços da ciência, mantendo-o tão distantes e alheios quanto a uma revolução de ponta 
em curso acelerado: nunca antes tivemos tantas informações científicas e equipamentos de concepção e construção complexas para acessá-las.

A maioria dos professores de Física do ensino médio não trabalha, durante o ano letivo, com Física Moderna ou, menos ainda, com Física Contemporânea. Há tempos repetimos sempre a mesma coisa e sempre do mesmo jeito. Precisamos construir e testar novos procedimentos pedagógicos para traçarmos um espaço-tempo escolar onde a Física de fronteira seja atuante no sentido de ser uma espécie de viga de sustentação intelectual para a edificação de estudantes críticos, contestadores da "verdade" atual e multiplicadores do conhecimento.

Por um lado, a Lei das Diretrizes e Bases da Educação (LDBE) preconiza que o professor deve oportunizar aos alunos do ensino médio tópicos de Física Moderna e Contemporânea.

Por outro, a escolha do assunto tratado nesse artigo se deve ao fato de que todas as pessoas, isto é, estudantes formais, informais ou indivíduos inteiramente leigos, em algum momento de suas vidas se perguntaram: Qual a natureza de nosso Universo? Do que o Universo é feito? [1]. Os estudantes do ensino médio precisam saber que o Universo (tudo que existiu, existe e existirá) é formado por grãos e que nós seres humanos somos formados, em última análise, por quarks up, quarks down e elétrons e que tudo isso é governado, mantido em funcionamento, até onde sabemos, por quatro interações fundamentais. Também é igualmente importante que saibam que além de outras propriedades intrigantes, os Neutrinos são como sondas pré-históricas que nos informaria sobre as condições físicas reinantes no Universo primordial. Ou como o Universo era três minutos após o Big-Bang.

Ressalto a importância da multifacetada Física Contemporânea, e suas consequências científicas e tecnológicas na vida cotidiana dos estudantes do ensino médio, visivelmente subliminar nos dizeres da Professora Iramaia [2]: "Estamos na era da informação, da alta densidade de fluxo de comunicação e sempre que nos comunicamos sobre um tema, valorizamos esse tema".

A importância da introdução da Física Contemporânea nas salas de aula do ensino médio está muito bem exposta qualitativamente em TERRAZZAN [3]; CAVALCANTE e TAVOLARO [4]; SOARES et. al. [5]; OSTERMANN [6]; MOREIRA $[7,8]$.

Nesse trabalho o estudo dos neutrinos é efetuado de modo a nortear a implementação do tema Física das Partículas, nas salas de aula do ensino médio, mediante a elaboração um produto educacional na forma de uma sequência didática (SD) sustentada por uma transcrição da Aprendizagem Significativa de Ausubel proposta por Moreira $[9,10]$ com o emprego da Unidade de Ensino Potencialmente Significativa (UEPS) [11].

O objetivo da Física das Partículas é estudar os constituintes fundamentais (sem estrutura interna/indivisível) da matéria, suas propriedades e suas interações. Para isso foi elaborado o Modelo Padrão de Física das Partículas [7]. Trata-se do mais complexo e sofisticado aparato físico-matemático em construção produzido pela espécie humana. Todavia, não explica a gravidade, a matéria escura e a energia escura. Atualmente sabemos que toda a matéria bariônica é formada por 61 partículas elementares.

Até onde conhecemos, as partículas fundamentais são formadas por seis quarks (up, down, estranho, charme, bottom e top), três léptons carregados (elétron, muon e tau) com seus três neutrinos associados.

O conceito de força em Física está associado à troca de partículas: partículas são emitidas (geradas) e absorvidas (destruídas). Essas partículas são chamadas de portadoras de forças: os fótons veiculam a força eletromagnética; os bósos $W^{ \pm}$e o $Z^{0}$ a força fraca; e os glúons, por sua vez, transportam a força forte. Todos os léptons interagem por meio da força fraca, mas somente os quarks sentem a força fraca e as partículas carregadas sentem a força eletromagnética.

Os neutrinos são partículas elementares produzidas por reações nucleares ou decaimento beta (duplo beta também?); têm carga elétrica nula; spin $1 \frac{1}{2}$ (férmions); e massa não nula [5,6]. De um lado, os neutrinos de baixa energia têm origem no sol e outros eventos similares. De outro, os neutrinos de alta energia são formados por eventos cósmicos extremos, tais como buracos negros e suas fusões, supernovas e Big-Bang. Foi proposto por Pauli em 1930 para tentar desesperadamente salvar o teorema da conservação da energia e do momento linear. Trata-se de uma partícula difícil de ser aprisionada: 02 colisões a cada bilhão de neutrinos que passam pelos detectores.

Supunha-se que os neutrinos eram partículas sem massa até a descoberta do processo de oscilação dos neutrinos. Esse fenômeno indica que os neutrinos têm massa não nula e os estados próprios de massa $\left(v_{1}, v_{2}, v_{3}\right)$ são composições de seus próprios sabores $\left(v_{e}, v_{\mu}, v_{\tau}\right)[13]$.

Algumas aplicações dos neutrinos: Monitoramento de proliferação nuclear; computação quântica; tele comunicações com NU's; prospecção mineral e de petróleo; grandes estruturas de telescópios a NU's como instrumentação oceanográfica, geofísica e 
biológica em águas profundas: monitoramento em tempo real; TÉLESCOPE À NEUTRINOS KM3NET ( abrindo uma nova janela sobre nosso universo); utilização de neutrinos atmosféricos de alta energia ou feixes de neutrinos artificiais para realizar uma tomografia da Terra, ou seja, um mapa da densidade de matéria no interior da Terra.

Conforme Dirac toda partícula (férmion) tem sua artipartícula. Mas de acordo com o físico Etorre Majorana o neutrino é sua própria antipapartícaula [14]. Hà uma experiência em curso tentando demonstrar o duplo decaimento [15] . Caso a existência desse tipo de decaimento seja evidenciado experimentalmente, o neutrino será sua própria antipartícula. Isso poderia explicar por que existe mais matéria que antimatéria no Univesro, ou seja, por que existimos !

\section{Metodologia}

2.1 Apresentações da proposta do produto

O produto educacional final almejado na investigação em questão deverá facilitar a priori o ensino de Física contemporânea a alunos do ensino médio via uma SD na forma de uma transcrição da Aprendizagem Significativa de Ausubel. Acredito que o produto poderá tornar as aulas de Física menos mecanicistas, menos livrescas, ou seja, tornar-se uma ferramenta de ensino oposta àquela tradicional utilizada no ensino bancário, tradicional de Física.

A ambientação das aulas será, sobretudo no laboratório de informática, sala de aula e biblioteca. As aulas constarão de grupos de discussões, aulas expositivas dialogadas e simulações computacionais. O tópico preferencialmente abordado será sobre neutrinos. Os materiais utilizados pelos alunos serão textos, vídeos e aplicativos de física. Quanto aos recursos educacionais podemos utilizar quadro negro, giz, computador, televisão e Datashow.

\subsection{Principais resultados esperados}

Produzir um produto educacional como sequência didática relativa aos temas Física de Partículas e Neutrinos para o ensino médio (enfatizando a sua importância para a compreensão do Universo), que permita introduzir conceitos de Física Contemporânea de forma clara e objetiva. Espera-se ainda despertar o interesse na disciplina Física pelos estudantes através deste tema, o que pode contribuir para uma diminuição nos índices de reprovação em Física.

\section{3- Construindo uma UEPS sobre Física de partículas com foco especial nos Neutrinos}

A seguir apresentamos as etapas da UEPS que utilizamos na construção da SD cuja característica principal no nosso entendimento é o aumento progressivo da complexidade das atividades em estrita conformidade com aquilo que é preconizado por Moreira em [11]. Vamos lá então:

Aula 01- Definir o tópico específico a ser abordado Temas: Modelo Padrão de Física das Partículas e Neutrinos

Aula 02- Criar/propor situações que levem os estudantes a externalizarem seu conhecimento prévio aceito ou não aceito no contexto do assunto abordado. -Atividade: Avaliação individual

-Descrição: O professor entregará aos estudantes um questionário com as seguintes perguntas: O que são átomos? O que tem dentro deles? Qual a carga do elétron, do próton e do nêutron? Existe alguma coisa imediatamente próxima ao seu redor? Se sim, o quê? Se prótons repelem prótons, por que o átomo não desmancha? Por que o elétron não cai sobre o núcleo? Quais são as quatro interações (forças) da natureza? Já ouviram falar de neutrinos? Logo em seguida o Professor recolherá o material para análise das respostas e reproduzirá o vídeo [16].

Aula 03- Propor situações-problema, em nível bem introdutório, levando em conta o conhecimento prévio do aluno.

-Atividade: Leituras em casa

Descrição: O professor entregará aos estudantes os textos $[17,18,19]$ e as dúvidas deverão ser apresentadas ao Professor antes da próxima aula. De posse dessas dúvidas os professor construirá slides para serem empregados no método IpC [20].

Aula 04- Aprofundando o conhecimento

Atividade: Leitura em casa

Descrição: O professor entregará aos estudantes os textos [21, 22, 23], solicitando-os também para assistir os vídeos [24,25]. As dúvidas deverão ser apresentadas ao Professor antes da próxima aula.

De posse dessas dúvidas os professor construirá novamente slides para serem empregados no método IpC [ 20].

Aula 05- Nova situação-problema

Atividade: Simulação computacional sobre decaimento beta [26].

Descrição: O professor levará os alunos para o laboratório de informática onde realizarão a simulação sobre emissão de neutrino.

Aula 06: Observando a diferenciação progressiva via aula dialogada interativa

A principal meta da SD sob a forma de uma UEPS é fazer com que o sistema cognitivo dos alunos adquira progressivamente conhecimentos novos, ou melhor, 
que conhecimentos mais simples permitam a compreensão progressiva de conhecimentos mais elaborados.

O professor poderá promover debates individuais ou em equipes com o intuito de verificar se os estudantes sabem explicar o que é, por exemplo, uma molécula; um átomo; uma partícula; o modelo padrão e para que serve; o que são neutrinos e etc. Em suma: se os estudantes são capazes de perceber que há um processo natural que constrói tudo que existe via interações entre partículas e campos. O professor deverá observar se houve de fato indícios de aprendizagem significativa.

Aula 07-Avaliação Somativa da UEPS durante todo o processo de aplicação

Atividade: Avaliação-questionário tradicional

Descrição: O professor aplicará os questionários individuais [27-34] sobre Modelo Padrão de Física das Partículas e Neutrinos e durante a correção deverá observar se houve de fato indícios de aprendizagem significativa.

\section{Aula 08-Avaliação da UEPS}

Atividade: Aplicação de questionário de opinião Descrição: o Professor deverá aplicar um questionário escrito aos estudantes sobre aspectos da metodologia empregada. Uma sugestão interessante é um questionário oral com a turma com o objetivo de promover a troca de significados. Estão de posse dessas duas fontes de dados, o professor poderá otimizar sua UEPS, pois nada em educação está pronto, acabada. Tudo pode sofrer inovaçãoes.

\section{Aula 09 - Avaliação da UEPS}

Atividade: Aplicação de questionário de opinião

Descrição: o Professor deverá elaborar e aplicar um questionário escrito aos estudantes sobre aspectos da metodologia empregada. Uma sugestão interessante é um questionário oral com a turma com o objetivo de promover a troca de significados. Estão de posse dessas duas fontes de dados, o professor poderá otimizar sua UEPS, pois nada em educação está pronto, acabada. Tudo pode sofrer inovaçãoes.

\section{Considerações Finais}

Após a aplicação do produto educacional será feita uma análise minuciosa dos resultados das avaliações objetivando a detecção de vestígios de aprendizagem significativa. Também os estudantes serão consultados sobre a eficiência do produto educacional e se têm sugestões para serem inseridas no produto educacional.

\section{Agradecimentos}

Ao Professore Flávio Gimenes e a todos os professores e funcionários do MNPEF/UFES

\section{Referências}

[1] Cern (Conseil Européen pour la Recherche Nucléaire).Disponível em < https://home.cern/>. Acesso em: 18 Dez. 22020.

[2] DE PAULO, I.J.C. O TEXTO DIDÁTICO DE APOIO COMO UM PRODUTO EDUCACIONAL-UMA OPÇÃO VIÁVEL. Coordenadora CPG Nacional MNPEF-SBF.

[3] TERRAZZAN, E. A.. Inserção de física moderna e contemporânea no ensino de física na escola de $2^{\circ}$ Grau. Caderno Catarinense de Ensino de Física, Florianópolis, SC, v. 9, n. 3, p. 209-214, 1992.

[4] CAVAlCANTE, M. A.; Tavolaro, C. R. C. Uma oficina de física moderna que vise a inserção no ensino médio. Caderno Catarinense de Ensino de Física, Florianópolis, SC, v. 18, n. 3, p. 298-316, 2001.

[5] SOARES, T.C; JÚNIOR, H.B; HELAYEL-NETO, J.A.; SANTOS, A, (ilustração). Física de Partículas vista pelas Interações Fundamentais e Formação de Professores. Editora São Paulo: Editora Livraria de Física, 2018.

[6] OSTERMANN, F. Um Texto para Professores do Ensino Médio sobre Partículas Elementares. Revista Brasileira de Ensino de Física, vol. 21, no. 3, Setembro, 1999. UFRS, Instituto de Física/Porto Alegre - RS Brasil.

[7] MOREIRA, Marco Antônio. O Modelo Padrão da Física de Partículas. Revista Brasileira de Ensino de Física, v. 31, n. 1, 1306 (2009).

[8] MOREIRA, Marco Antônio. Partículas e interações Física na Escola, v. 5, n. 2, 2004.

[9] MOREIRA, Marco A. (2006). A Teoria da aprendizagem Significativa e sua implementação em sala de aula. Brasília: Editora Universidade de Brasília.

[10] Moreira, Marco A. O QUE É AFINAL APRENDIZAGEM SIGNIFICATIVA? (After all, what is meaningful learning?)

Instituto de Física - UFRGS

Porto Alegre -RS

[11] MOREIRA, Marco A. (2006). UNIDADES DE ENSINO POTENCIALMENTE SIGNIFICATIVAS UEPS* Potentially Meaningful Teaching Units - PMTU. Instituto de Física - UFRGS -Porto Alegre-RS.

[12] ÉBOLI, J. P. Partículas Elementares Oscar. Instituto de Física - USP.

[13] The Double Chooz Collaboration., de Kerret, H., Abrahão, T. et al. Double Chooz $\boldsymbol{\theta}_{13}$ measurement via total neutron capture detection. Nat. Phys. 16, 558564 (2020).

Disponível em <https://doi.org/10.1038/s41567-0200831-y>. Acesso em: 23 Dez. 2020. 
[14] Website FERMILAB. Neutrinos: Majorana or Dirac? Disponível em

$<$ https://news.fnal.gov/2012/09/neutrinos-majorana-ordirac/>. Acesso em: 15 Jul. 2020.

\section{[15] T2K Experiment.}

Disponível em: <https://t2k-experiment.org/2020/04/t2kresults-restrict-possible-values-of-neutrino-cp-phase/>. Acesso em: 24 de Jul. 2020.

[16] CERN. Voyage into the world of atoms. Disponível em <https://videos.cern.ch/record/2307615> Acesso em: 22 de Dez. 2020.

[17] Revista Brasileira de Ensino de Física, v.31, n.1, 1306 (2009). O Modelo Padrão da Física de Partículas. Disponível em <http://moreira.if.ufrgs.br/modelopadrao.pdf> Acesso em: 24 Jul. 2020.

[18] Física na Escola, v. 5, n. 2, 2004. Partículas e interações. Disponível em<http://www.sbfisica.org.br/fne/Vol5/Num2/v5n1a03. pdf>. Acesso em: 24 de jul. 2020.

[19] Symmetry: dimentions of the particles. The particle physicsof you.

Disponível em

$<$ https://translate.google.com/translate?hl=pt-

BR\&sl=en\&u=https://www.symmetrymagazine.org/articl e/the-particle-physics-of-you\&prev=search>

Acesso em: 24 de Jul. 2020.

[20] ARAÚJO, I.S; MAZUR, E. INSTRUÇÃO PELOS COLEGAS E ENSINO SOB MEDIDA: UMA PROPOSTA PARA O ENGAJAMENTO DOS ALUNOS NO PROCESSO DE ENSINOAPRENDIZAGEM

DE FísICA. Instituto de Física - UFRGS Porto Alegre RS e Harvard University Cambridge - MassachusettsEUA.

[21] Revista Um olhar para o futuro Neutrinos as misteriosas partículas-fantasmas misteriosas partículas-fantasma. Instituto de Física Gleb Wataghin/Universidade Estadual de Campinas). [22] MacTutor History of Mathematics Archive:

Wolfgang Ernst Pauli.

Disponível em <https://mathshistory.standrews.ac.uk/Biographies/Pauli/> Acesso em: 25 de Jul. 2020.

[23] Centro Brasileiro de Pesquisas Físicas (CBPF). Ettore Majorana: $O$ Drama de Consciência de um Jovem Cientista.Disponívem em:

http://www.cbpf.br/ cirto/MecEstNaoExten_HTML/AUL AS/Aula_03/Morcelle_\&_Campbell_\&_Tavares_\&_Vug man_\%28Ettore_Majorana_O_Drama_De_Consciencia
_De_Um_Jovem_Cientista\%29_\%5BCBPF_Ciencia_E

_Sociedade_2006\%5D.pdf

Acesso em: 20 Dez. 2020

[24] Física em Casa

Neutrinos: $O$ que são e para que servem?

Disponível em:

https://www.youtube.com/watch?v=2K5GWlySpTA

Acesso em: 22 Dez. 2020.

[25] Física em Casa

Oscilações quânticas de neutrinos - Marcelo Guzzo

\& Marina Reggiani-Guzzo

Disponível em

<https://www.youtube.com/watch?v=Ke5CjfDUy

Ps>. Acesso em: 22 Dez. 2020.

[26] Simulador PHET - Colorado

Simulação computacional sobre decaimento beta.

Disponível em

$<$ https://phet.colorado.edu/sims/cheerpj/nuclear-

physics/latest/nuclear-physics.html?simulation=betadecay\&locale=pt_BR>

Acesso: em: 22 de Dez.de 2020.

\section{[27] EXERCÍCIOS SOBRE PARTÍCULAS DE UM} ÁTOMO.

Disponível em

$<$ https://exercicios.brasilescola.uol.com.br/exerciciosquimica/exercicios-sobre-particulas-um-atomo.htm>. Acesso em: 20 Dez. 2020.

[28,29,30,31] EXERCÍCIOS SOBRE MODELOPADRÃO DA FÍSICA DE PARTÍCULAS.

Disponível em

$<$ https://exercicios.brasilescola.uol.com.br/exercicios-

fisica/exercicios-sobre-modelo-padrao-fisica-

particulas.htm\#: :text=Os\%20dois\%20grupos\%20princi pais\%20do,f\%C3\%A9rmions\%20s\%C3\%A3o\%20a\%20 pr\%C3\%B3pria\%20mat\%C3\%A9ria>.

Acesso em: 20 Dez. 2020.

\section{[32] Questões de Concursos}

Disponível em <https://www.qconcursos.com/questoesde-vestibular/questoes/4b5df869-30>.

Acesso em: 20 Dez 2020.

\section{[33,34] EXERCÍCIOS SOBRE FÍSICA DE}

PARTÍCULAS.

Disponível em

$<$ https://exercicios.brasilescola.uol.com.br/exerciciosfisica/exercicios-sobre-fisica-

particulas.htm\#: :text=Quest\%C3\%A30\%202,recebem \%200\%20nome\%20de\%20b\%C3\%B3sons..

Acesso em:20 Dez. 2020; 